\title{
Association Between Statin Medications and COPD-Specific Outcomes: A Real-World Observational Study
}

\author{
Mayank Ajmera $^{1} \cdot$ Chan Shen $^{2} \cdot$ Usha Sambamoorthi $^{3}$
}

Published online: 10 December 2016

(C) The Author(s) 2016. This article is published with open access at Springerlink.com

\begin{abstract}
Background Disease-modifying drugs are not yet available for the management of chronic obstructive pulmonary disease (COPD). HMG-CoA reductase inhibitors (statins) have anti-inflammatory properties and are therefore being considered for use in the management of COPD.

Objective Our objective was to examine the association between statin use and COPD-specific outcomes in a realworld setting.

Methods This was a retrospective longitudinal dynamic cohort study that used Medicaid claims data from multiple years (2005-2008) to identify patients with newly diagnosed COPD. Statin therapy was determined from the prescription drug file using National Drug Codes (NDCs). COPD-specific outcomes such as hospitalizations and emergency room and outpatient visits were identified based on a primary diagnosis of COPD. Multivariable logistic regressions with inverse probability treatment weights (IPTWs) were used to examine the relationship between statin therapy and COPD-specific outcomes.

Results The study included 19,060 Medicaid beneficiaries with newly diagnosed COPD, $30.3 \%$ of whom received statins during the baseline period. Adults who
\end{abstract}

Mayank Ajmera

majmera@rti.org

1 RTI Health Solutions, 300 Park Offices Drive, Research Triangle Park, Durham, NC, USA

2 Department of Biostatistics and Health Services Research, MD Anderson Cancer Center, University of Texas, Houston, TX, USA

3 Department of Pharmaceutical Systems and Policy, School of Pharmacy, West Virginia University, Morgantown, WV, USA received statins had significantly lower rates of COPDspecific hospitalizations ( 4.7 vs. $5.2 \% ; p<0.05$ ), emergency room visits $(13.4$ vs. $15.4 \% ; p<0.001)$, and outpatient visits $(41.4$ vs. $44.7 \% ; p<0.001)$ than those who did not receive statin therapy. Even after adjusting for observed selection bias with IPTWs, adults receiving statins were less likely to have COPD-specific hospitalizations [adjusted odds ratio (AOR) 0.76; 95\% confidence interval (CI) 0.66-0.87], emergency room visits (AOR $0.81 ; 95 \%$ CI $0.75-0.89$ ), and outpatient visits (AOR 0.86; 95\% CI 0.80-0.91) than those not receiving statins.

Conclusions Findings from this study suggest statins have beneficial effects in patients with newly diagnosed COPD and warrant further clinical trial investigation.

\section{Key Points}

This retrospective study using data from Medicaid analytic extracts found the use of HMG-CoA inhibitors (statins) among beneficiaries with newly diagnosed chronic obstructive pulmonary disease (COPD) may be associated with lower rates of hospitalization and fewer emergency room and outpatient visits.

Findings from the multivariable logistic regression also showed that statin use was associated with a lower likelihood of COPD-specific healthcare resource use.

These study findings suggest improved outcomes among statin users with COPD and thus warrant further clinical trial investigation. 


\section{Introduction}

Treatment for chronic obstructive pulmonary disease (COPD) currently focuses on reducing the frequency of acute exacerbations and relieving the symptoms of COPD, such as shortness of breath, through acute and maintenance pharmacologic therapies and pulmonary rehabilitation [1]. To date, no therapeutic options that alter the prognosis of COPD (also known as disease-modifying drugs) have been established. The pathophysiology of the disease revolves around local inflammation in the lungs [1]. However, emerging understanding of COPD suggests the presence of systemic inflammation among individuals with COPD [2-4]. It was recently documented that individuals with COPD are more likely to have certain chronic conditions with common inflammatory pathways than individuals without COPD. These conditions include depression (20.6 vs. 12.5\%), coronary heart disease (12.7 vs. $6.1 \%$ ), congestive heart failure (12.1 vs. $3.9 \%)$, and stroke (8.9 vs. $4.6 \%$ ) [5]. Unfortunately, the current acute and maintenance pharmacologic therapies for the management of COPD do not specifically target this systemic inflammation $[2,6]$. Thus, researchers have been exploring the beneficial effects of other medications that may lead to a reduction in systemic inflammation among individuals with COPD [6-8].

\subsection{Statins, Anti-Inflammatory Properties, and Improved Outcomes Among Individuals with Chronic Obstructive Pulmonary Disease (COPD)}

HMG-CoA inhibitors (statins) are a class of cholesterollowering drugs that have demonstrated effectiveness in the prevention and management of cardiovascular diseases [9]. Schönbeck and Libby [10] reviewed in vitro and in vivo studies and concluded that statins also have anti-inflammatory properties. Several studies have shown statins to be of benefit in reducing inflammatory biomarkers. Lee et al. [11] found that smoking-related inflammation in the lungs of rats was alleviated by a lipophilic statin (simvastatin). In humans, a randomized controlled trial (RCT) showed that pravastatin (hydrophilic statin) significantly reduced levels of inflammatory biomarkers [12]. This evidence is also supported by preclinical models that have revealed the pleiotropic/antiinflammatory therapeutic benefits attributable to statins [13].

Documented evidence of the beneficial effects of statins on outcomes among individuals with COPD also exists. One randomized trial [12] and some observational studies [14-18] have examined the role of statins in the management of COPD. Most of these studies have provided evidence of the beneficial effects of statins in improving lung function [14] and exercise time as a measure of health status [12] and in reducing COPD exacerbations [16] and all-cause [15, 19-21] and COPD-specific mortality [19]. However, only a handful of studies have examined the effects of statins on COPD-specific clinical outcomes such as hospitalizations in a real-world practice setting.

A time-matched nested case-control study on the effects of statins in elderly individuals with COPD indicated that, compared with no statin use, any statin use was associated with a reduced likelihood of COPD-related hospitalization irrespective of their cardiovascular risk [high cardiovascular risk: risk ratio (RR) $0.71 ; 95 \%$ confidence interval (CI) 0.56-0.91; low cardiovascular risk: RR 0.71; 95\% CI 0.64-0.77] [20]. These findings were similar to those from a nationwide cohort study in Taiwan [17] reporting that, among 6252 individuals with newly diagnosed COPD, statin use significantly decreased the hazard ratio (HR) of COPD-related hospitalization (HR $0.66 ; 95 \%$ CI 0.60-0.74) compared with no statin use.

These studies have several limitations that restrict our ability to better understand the relationship between statin therapy and COPD-specific health outcomes. Keddissi et al. [14] assessed the impact of statin use among veterans with COPD but had a limited sample size of only 418 individuals and only represented hospitalized patients who were either former or current smokers. The other studies that evaluated the use of statins for improving COPDspecific outcomes had other issues in terms of generalizability to the general US population. For example, some papers were based on non-US populations [17, 20], whereas others were conducted among specific subgroups such as those with COPD and cardiovascular risk [20].

Therefore, the primary objective of this study was to evaluate the relationship between statins and COPDspecific outcomes such as hospitalizations and emergency room and outpatient visits in a US population seeking care in real-world practice settings.

\section{Methods}

\subsection{Study Design}

This was a retrospective longitudinal dynamic cohort study to examine the relationship between statins and COPDspecific clinical outcomes. Two separate 2-year panels using data from multiple years (2005-2008) were created in which Medicaid beneficiaries with diagnosed COPD were identified in the years 2006 and 2007. We used the first service date with a COPD diagnosis as the index date. A detailed description of the procedure used to identify the study population is provided in the following sections. Two distinct study observation periods consisted of the baseline period (defined as 1 year before the index date) and follow- 
up period (defined as 1 year after the index date). The baseline period was used to assess statin use and other beneficiary characteristics, whereas the follow-up period was used to ascertain COPD-specific clinical outcomes.

\subsection{Data Source}

Medicaid analytic extract (MAX) files containing information on enrollment, inpatient visits, other therapy, and the use of prescription drugs were used [22]. The personal summary file provided information on eligibility, demographics (age, sex, and race), managed care enrollment, and utilization summary. The other four files contained information on fee-for-service claims for services used. Other therapy files included information regarding claims for Medicaid services provided at an outpatient level such as clinic, physician, laboratory, and home-health services. Hospitalization diagnosis codes, procedures, length of stay for each hospitalization, and expenditures related to hospitalizations were extracted from the inpatient files. National drug codes (NDCs) for prescription drugs, supply days, fill dates, and payments for prescription drugs were obtained from the prescription drug files.

We used data for beneficiaries residing in California (CA), Illinois (IL), New York (NY), and Texas (TX). These states were chosen to capture the diverse geographic and racial/ethnic populations represented by Medicaid.

\subsubsection{Area Resource File}

We also determined each Medicaid beneficiary's contextual county-level variables using the area resource file (ARF), a comprehensive county-level dataset compiled by the Health Resources \& Services Administration Bureau of Health Professions. It contains more than 6000 variables providing information about the nation's counties. The information contained within the ARF includes type of health facilities in the various counties, number and type of health professions, resource scarcity measures, health status, economic activity, health training programs, and socioeconomic and environmental characteristics. These files also include county codes and descriptors that enable linkage with several secondary datasets such as MAX. We used the county codes and state information to link MAX files with the 2005 ARF file.

\subsection{Study Population: Medicaid Beneficiaries with Newly Diagnosed COPD}

Medicaid beneficiaries with diagnosed COPD were identified using inpatient and outpatient visits (physician office visits and clinic services only). Medicaid beneficiaries with at least one inpatient visit or two outpatient visits 14 days apart (obtained using type of service codes) for COPD based on International Classification of Diseases, Ninth Revision, Clinical Modification (ICD-9-CM) codes for chronic bronchitis (491.xx), emphysema (492.xx), or unspecified chronic airway obstruction (496.xx) were considered to have diagnosed COPD. These diagnosis codes have been used in published research to identify COPD and evaluate medical treatment and health outcomes among individuals with COPD [23-25], and the sensitivity and specificity of using these codes to identify patients with COPD has been established [26, 27]. Gershon et al. [27] reported that identifying COPD using one or more ambulatory claims and/or one or more hospitalizations for COPD resulted in a sensitivity of $85.0 \%(95 \% \mathrm{CI}$ 77.0-91.0) and a specificity of 78.4\% (95\% CI 73.6-82.7). However, we used one inpatient claim or at least two outpatient claims to identify individuals with COPD to increase the specificity of the algorithm. We only included Medicaid beneficiaries with newly diagnosed COPD. To obtain the data for these individuals, we created a washout period (1 year prior to the index date of COPD diagnosis). Only Medicaid beneficiaries who did not have a COPD diagnosis in the washout period were considered to have newly diagnosed COPD and included in our study population.

Other inclusion criteria included (1) aged 40-64 years as of the index date (among young adults, this age group is at highest risk of COPD), (2) continuous eligibility during the baseline and follow-up periods, (3) no dual Medicaid/ Medicare coverage (dual eligibility represents high-cost and severely ill beneficiaries), (4) enrolled in fee-for-service plans throughout the study observation period, (5) alive during the study observation period, (6) and use of services (inpatient or outpatient).

\subsection{Dependent Variables: COPD-Specific Outcomes}

The following variables were identified as COPD-specific outcomes: (1) COPD-specific hospitalizations (yes/no), (2) COPD-specific emergency room visits (yes/no) obtained from inpatient and outpatient claims, and (3) COPDspecific outpatient visits (low and high) derived by categorizing the number of COPD-specific outpatient visits greater than or equal to the median and less than the median value. Any healthcare claim with a primary diagnosis of COPD identified using ICD-9-CM codes was considered within COPD-specific outcomes.

\subsection{Key Independent Variable: Statin Therapy (Yes/No)}

Statin therapy was identified in the baseline period using the NDCs. Any Medicaid beneficiary with at least one 
prescription of a statin during the baseline period was considered to be a statin therapy user.

\subsection{Other Independent Variables}

These variables included year of diagnosis (2006 vs. 2007), demographic characteristics such as sex (women, men), race (White, African American, other), age in years (40-49, 50-59, 60-64), poverty eligibility (yes, no), medical eligibility (yes, no), number of clinical conditions (none, 1-3, 4-6, >6), serious mental illness (yes, no), alcohol abuse (yes, no), substance abuse (yes, no), tobacco use (yes, no), and polypharmacy (fewer than ten drug classes, ten or more drug classes). We also controlled for county-level characteristics obtained from the ARF. Quartiles for density of above high school education, unemployment, poverty, primary care providers, and specialist care providers were created. The density of these county-level characteristics was calculated by dividing the total number of each characteristic by the total county population. This density was further converted into per 1000 people by multiplying 1000 with the density. Other ARF characteristics included pulmonologist density (high vs. low) and cardiologist density (high vs. low), among others. In addition to these variables, there is a possibility of bias in the relationship between statin therapy and COPD-specific outcomes because of variations in state policies. Therefore, we adjusted for fixed effects for state variations using a dummy variable for the states (CA, IL, NY, TX) in our analyses.

We also controlled for the presence of common multimorbidity by constructing binary indicator variables to indicate the presence of common inflammatory conditions, including arthritis, cardiovascular disease, depression, diabetes, hypertension, hyperlipidemia, and osteoporosis using ICD-9-CM codes. We further categorized the multimorbidity variable into a binary categorical variable (yes/no).

\subsection{Statistical Analyses}

\subsubsection{Bivariate Analyses}

Subgroup differences in statin therapy and duration of statin therapy were tested using Chi squared tests of independence, as were bivariate differences in COPDspecific healthcare outcomes between those receiving and not receiving statin therapy.

\subsubsection{Multivariate Analyses}

To account for selection bias due to observed differences in baseline characteristics among individuals with and without statin therapy, we used inverse probability of treatment weighting (IPTW). This allowed us to calculate weights that were essentially the probability of an individual receiving the treatment (i.e., exposure in a non-randomized study) conditional on their observed stable covariates. Higher weights were assigned to individuals who were under-represented and lower weights were assigned to those who were over represented. We adjusted for the probability weight that we obtained from the logistic/multinomial logistic regressions on statin therapy and duration of statin therapy, respectively, to control for the observed selection bias. This created a balance in terms of distribution of potential confounders across treatment levels. The literature [28] has shown this method performs better than matching-based methods when the prevalence of treatment is relatively not extreme, which is the case in our study cohort $(30 \%)$.

We used logistic regressions and multinomial logistic regressions to examine the factors associated with any statin and long-term statin therapy, respectively. Multivariable logistic regressions with and without IPTW adjustment were used to determine the relationship between statin therapy and COPD-specific outcomes.

We used logistic regression to analyze the relationship between statin therapy, presence of multimorbidity, and COPD-specific healthcare outcomes. We created a variable with four categories: (1) statin therapy and multimorbidity, (2) no statin therapy and multimorbidity, (3) statin therapy and no multimorbidity, and (4) no statin therapy and multimorbidity. All analyses were conducted using SAS 9.3 (SAS Institute, Cary, NC, USA).

\section{Results}

After applying the eligibility criteria as shown in Fig. 1, the analytic cohort included 19,060 Medicaid beneficiaries with newly diagnosed COPD. Any statin therapy was observed in $30.3 \%$ ( $n=5771)$ of the Medicaid beneficiaries, three-quarters of whom received long-term statin therapy, defined as $\geq 120$ days of statin medication supply.

\subsection{Factors Associated with Statin Therapy}

Several demographic and clinical factors were associated with statin therapy and duration of statin therapy among Medicaid beneficiaries with newly diagnosed COPD (Table 1). We found significant differences between statin users and non-users, so we performed a multivariable logistic regression that adjusted for multimorbidity, number of other clinical conditions, year of observation, sex, race/ ethnicity, age, state, serious mental illness, alcohol use, substance use, and polypharmacy. This regression was used to calculate the IPTWs. We checked the balance of the explanatory variables before and after IPTW adjustment and 
Fig. 1 Selection of study population

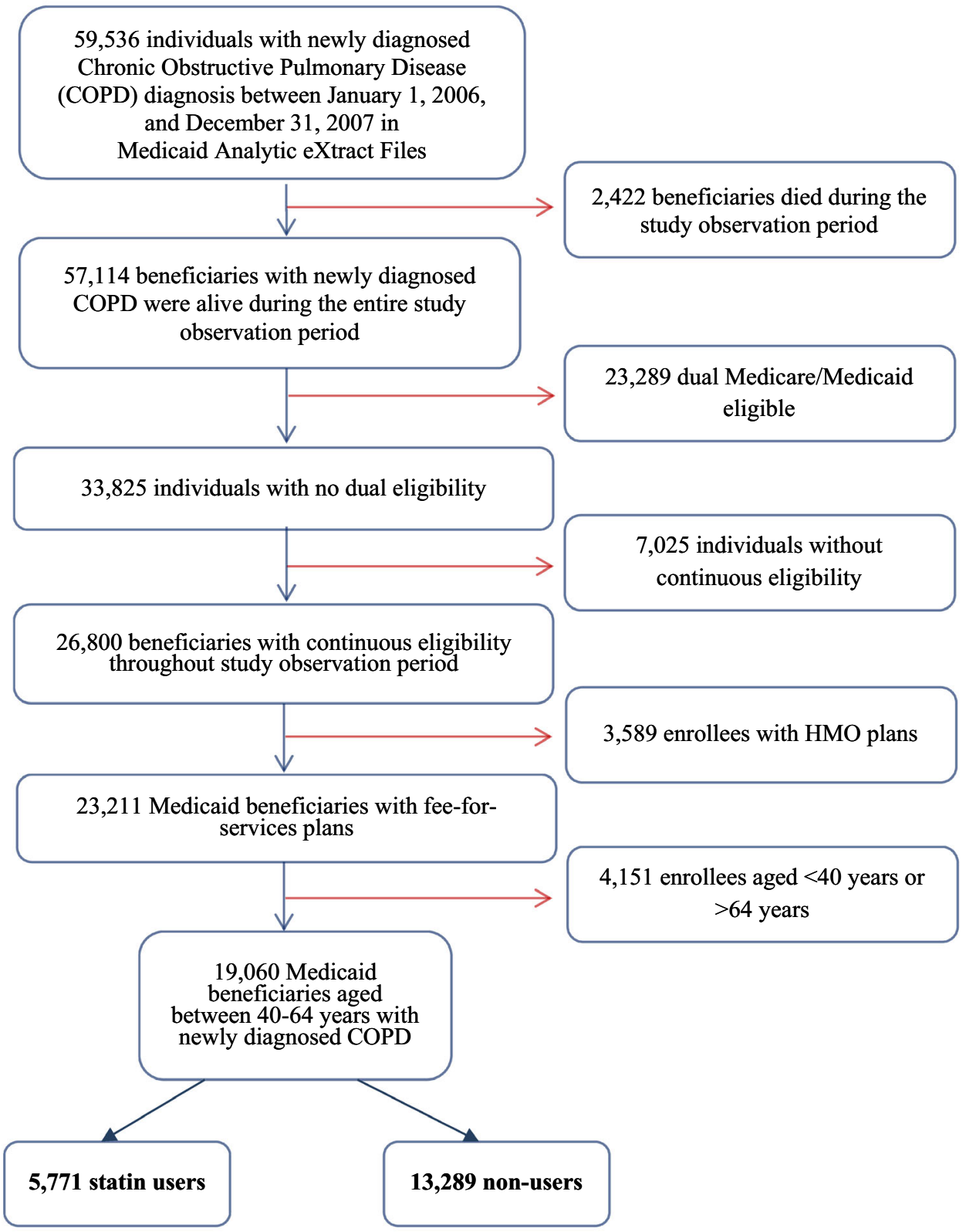

59,536 individuals with newly diagnosed

Chronic Obstructive Pulmonary Disease

uary 1,2006

Medicaid Analytic eXtract Files

7,114 beneficiaries with newly diagnosed observation period

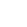

,800 beneficiaries with continuous eligibility

3,589 enrollees with HMO plans services plans

13,289 non-users observed that the group differences in statin use were no longer significant after IPTW adjustment (Table 1).

\subsection{Statin Therapy and COPD-Specific Outcomes}

As shown in Table 2, individuals receiving statin therapy had significantly lower rates of COPD-specific hospitalizations (4.7 vs. 5.2\%; $p<0.05)$, COPD-specific emergency room visits (13.4 vs. $15.4 \% ; p<0.001$ ), and COPDspecific outpatient visits $(41.4$ vs. $44.7 \% ; p<0.001)$ than adults who were not receiving statin therapy. Similarly, beneficiaries with any duration of statin therapy (long-term or short-term) had lower rates of COPD-specific healthcare utilization. The reduction in COPD-specific healthcare utilization was also observed even after controlling for baseline characteristics. Beneficiaries receiving statin therapy were $16 \%$ less likely to have COPD-specific hospitalization [adjusted odds ratio (AOR) $0.84 ; 95 \% \mathrm{CI}$ $0.71-0.98$ ], $11 \%$ less likely to have COPD-specific emergency room visits (AOR 0.89; 95\% CI 0.81-0.99), and $14 \%$ less likely to have COPD-specific outpatient visits (AOR 0.86; 95\% CI 0.80-0.92). To control for selection bias in observed variables, we conducted the same regression analyses after weighting with IPTWs obtained from logistic and multinomial logistic regressions on statin therapy and duration of statin therapy, respectively. These treatment weights were adjusted for the number of individuals in each category of statin therapy and duration of 
Table 1 Patient characteristics by statin (pre- and post- inverse probability treatment weighting)

\begin{tabular}{|c|c|c|c|c|c|c|c|c|}
\hline \multirow[t]{3}{*}{ Characteristic } & \multicolumn{5}{|c|}{ Pre-inverse probability treatment weighting } & \multicolumn{3}{|c|}{ Post-inverse probability treatment weighting } \\
\hline & \multicolumn{2}{|c|}{ Statin use } & \multicolumn{2}{|c|}{ No statin use } & \multirow[t]{2}{*}{$p$ value } & \multirow{2}{*}{$\begin{array}{l}\text { Statin use } \\
\text { Row (wt\%) }\end{array}$} & \multirow{2}{*}{$\begin{array}{l}\text { No statin use } \\
\text { Row (wt\%) }\end{array}$} & \multirow[t]{2}{*}{$p$ value } \\
\hline & $N$ & Row (\%) & $N$ & Row (\%) & & & & \\
\hline Total & 5771 & 30.1 & 13,289 & 69.9 & & & & \\
\hline Multimorbidity categories & & & & & $<0.0001$ & & & 0.887 \\
\hline Physical only & 3639 & 39.1 & 5668 & 60.9 & & 30.3 & 69.7 & \\
\hline Mental only & 184 & 14.3 & 1099 & 85.7 & & 31.3 & 68.7 & \\
\hline Both & 1345 & 36.0 & 2388 & 64.0 & & 30.3 & 69.7 & \\
\hline None & 603 & 12.7 & 4134 & 87.3 & & 30.2 & 69.8 & \\
\hline Cohort year & & & & & 0.007 & & & 0.436 \\
\hline $2005-2007$ & 3161 & 29.5 & 7560 & 70.5 & & 30.5 & 69.5 & \\
\hline $2006-2008$ & 2610 & 31.3 & 5729 & 68.7 & & 30.0 & 70.0 & \\
\hline Sex & & & & & 0.000 & & & 0.421 \\
\hline Women & 3646 & 32.2 & 7688 & 67.8 & & 30.5 & 69.5 & \\
\hline Men & 2125 & 27.5 & 5601 & 72.5 & & 30.0 & 70.0 & \\
\hline Age (years) & & & & & $<0.0001$ & & & 0.838 \\
\hline $40-49$ & 1386 & 21.8 & 4958 & 78.2 & & 30.6 & 69.4 & \\
\hline $50-59$ & 2942 & 31.9 & 6292 & 68.1 & & 30.1 & 69.9 & \\
\hline $60-64$ & 1443 & 41.4 & 2039 & 58.6 & & 30.3 & 69.7 & \\
\hline Race/ethnicity & & & & & $<0.0001$ & & & 0.836 \\
\hline White & 2935 & 31.7 & 6337 & 68.3 & & 30.1 & 69.9 & \\
\hline African-American & 1231 & 24.2 & 3854 & 75.8 & & 30.4 & 69.6 & \\
\hline Others & 1605 & 34.1 & 3098 & 65.9 & & 30.6 & 69.4 & \\
\hline State & & & & & $<0.0001$ & & & 0.791 \\
\hline California & 3179 & 30.6 & 7218 & 69.4 & & 30.5 & 69.5 & \\
\hline Illinois & 1257 & 30.6 & 2853 & 69.4 & & 29.7 & 70.3 & \\
\hline New York & 854 & 32.7 & 1761 & 67.3 & & 30.3 & 69.7 & \\
\hline Texas & 481 & 24.8 & 1457 & 75.2 & & 30.8 & 69.2 & \\
\hline Number of clinical conditions & & & & & $<0.0001$ & & & 0.961 \\
\hline None & 74 & 15.6 & 399 & 84.4 & & 31.0 & 69.0 & \\
\hline $1-3$ & 835 & 23.5 & 2713 & 76.5 & & 30.0 & 70.0 & \\
\hline $4-6$ & 1268 & 28.3 & 3205 & 71.7 & & 30.5 & 69.5 & \\
\hline$>6$ & 3594 & 34.0 & 6972 & 66.0 & & 30.3 & 69.7 & \\
\hline SMI & & & & & $<0.0001$ & & & 0.045 \\
\hline Yes & 1299 & 26.2 & 3665 & 73.8 & & 31.4 & 68.6 & \\
\hline No & 4472 & 31.7 & 9624 & 68.3 & & 29.9 & 70.1 & \\
\hline Alcohol abuse & & & & & $<0.0001$ & & & 0.808 \\
\hline Yes & 318 & 17.4 & 1506 & 82.6 & & 30.6 & 69.4 & \\
\hline No & 5453 & 31.6 & 11,783 & 68.4 & & 30.3 & 69.7 & \\
\hline Substance abuse & & & & & $<0.0001$ & & & 0.272 \\
\hline Yes & 457 & 17.3 & 2183 & 82.7 & & 31.2 & 68.8 & \\
\hline No & 5314 & 32.4 & 11,106 & 67.6 & & 30.2 & 69.8 & \\
\hline Polypharmacy & & & & & $<0.0001$ & & & 0.515 \\
\hline Yes & 4361 & 44.2 & 5502 & 55.8 & & 30.5 & 69.5 & \\
\hline No & 1410 & 15.3 & 7787 & 84.7 & & 30.1 & 69.9 & \\
\hline
\end{tabular}

Based on 19,060 Medicaid Beneficiaries with newly diagnosed COPD obtained from Medicaid analytic extract files observed during 2005-2008. IPTWs were derived from a logistic regression on statin use with adjustments for all the variables included in the table. Row percentages reflect the percentage of non-users among subgroups with specific characteristics

COPD chronic obstructive pulmonary disease, IPTWs inverse probability treatment weights, SMI serious mental illness, Wt weighted 
Table 2 Logistic regressions on chronic obstructive pulmonary disease-specific healthcare utilization among Medicaid beneficiaries with newly diagnosed chronic obstructive pulmonary disease

\begin{tabular}{|c|c|c|c|c|c|c|}
\hline & $N$ & Row $(\%)$ & Significance & AOR & $95 \% \mathrm{CI}$ & Significance \\
\hline \multicolumn{7}{|c|}{ COPD-specific hospitalizations } \\
\hline Total & 998 & 5.2 & & & & \\
\hline Statin therapy & & & $*$ & & & \\
\hline Yes & 270 & 4.7 & & 0.84 & $0.71-0.98$ & $*$ \\
\hline No & 728 & 5.5 & & & & \\
\hline \multicolumn{7}{|c|}{ COPD-specific emergency room visits } \\
\hline Total & 2824 & 14.8 & & & & \\
\hline Statin therapy & & & $* * *$ & & & \\
\hline Yes & 775 & 13.4 & & 0.89 & $0.81-0.99$ & $*$ \\
\hline No & 2049 & 15.4 & & & & \\
\hline \multicolumn{7}{|c|}{ COPD-specific outpatient visits } \\
\hline Total & 7200 & 43.7 & & & & \\
\hline Statin therapy & & & $* * *$ & & & \\
\hline Yes & 2032 & 41.4 & & 0.86 & $0.80-0.92$ & $* * *$ \\
\hline No & 5168 & 44.7 & & & & \\
\hline
\end{tabular}

Based on 19,060 Medicaid beneficiaries with newly diagnosed COPD obtained from Medicaid analytic extract files observed during 2005-2008. Asterisks represent significant group differences in likelihood of COPD-specific healthcare utilization by statin therapy compared with the reference group (none) obtained from adjusted logistic regression analyses. Adjusted logistic regressions controlled for cohort year, sex, race, age, state, poverty eligibility, multimorbidity number of other clinical conditions, serious mental illness, alcohol abuse, substance abuse, tobacco use, polypharmacy, county-level variables, including above high school education density (quartiles), unemployment density (quartiles), poverty density (quartiles), metro status, primary care shortage area, mental health shortage area, primary care provider density (quartiles), hospital beds density (quartiles), psychiatric hospital, pulmonologist density and cardiologist density

$A O R$ adjusted odds ratio, $C I$ confidence interval, $C O P D$ chronic obstructive pulmonary disease

$* 0.01 \leq p<0.05$

$* * 0.001 \leq p<0.01$

$* * * p<0.001$

statin therapy. The results from these analyses are presented in Table 3. Even after using IPTW, we found that individuals receiving statin therapy were significantly less likely to have COPD-specific healthcare outcomes. In fact, the magnitude of reduction and significance strength was enhanced after controlling for selection bias using the IPTW technique.

\subsection{Statin Therapy, Multimorbidity, and COPD- Specific Healthcare Outcomes}

We analyzed the relationship between statin therapy and COPD-specific healthcare outcomes in the presence of multimorbidity and found that beneficiaries with multimorbidity receiving statin therapy were less likely to have COPD-specific healthcare outcomes than those with multimorbidity not receiving statin therapy. For example, beneficiaries with multimorbidity receiving statin therapy were $18 \%$ less likely to have COPD-specific hospitalization (AOR 0.82 ; 95\% CI 0.70-0.97) than those with multimorbidity not receiving statin therapy. Detailed results are presented in Table 4.

\subsection{Secondary Analyses: Duration of Statin Therapy (Long-Term/Short-Term/No Statin Therapy)}

Individuals receiving long-term statin therapy were differentiated from those receiving short-term statin therapy. Medicaid beneficiaries with newly diagnosed COPD with $\geq 120$ days of statin supply during the baseline period were considered to be receiving long-term therapy. Individuals with $<120$ days of statin supply were categorized as receiving short-term statin therapy, and those without any statin claim were grouped under 'no statin' therapy.

Analyses of duration of statin therapy revealed that only long-term statin therapy was associated with lower COPDspecific healthcare outcomes. Beneficiaries receiving longterm statin therapy were less likely to have COPD-specific hospitalizations (AOR 0.81; 95\% CI 0.71-0.99), COPDspecific emergency room visits (AOR 0.86; 95\% CI 0.77-0.96), and COPD-specific outpatient visits (AOR 0.84; 95\% CI 0.78-0.92) (data not represented in tabular form).

Moreover, after using IPTW, short-term statin therapy also showed significant reductions in COPD-specific 
Table 3 Logistic regressions on chronic obstructive pulmonary disease-specific healthcare utilization among Medicaid beneficiaries with newly diagnosed chronic obstructive pulmonary disease ( $n=19,060)$; inverse probability treatment weight adjustment

\begin{tabular}{llll}
\hline & AOR & $95 \%$ CI & Significance \\
\hline $\begin{array}{l}\text { COPD-specific hospitalizations } \\
\text { Statin therapy }\end{array}$ & & & \\
$\quad$ Yes & 0.79 & $0.68-0.92$ & $* *$ \\
$\quad$ No & & & \\
COPD-specific emergency room visits & & \\
$\quad$ Statin therapy & & & \\
$\quad$ Yes & 0.79 & $0.68-0.92$ & $* * *$ \\
$\quad$ No & & & \\
COPD-specific outpatient visits & & & \\
Statin therapy & & & \\
$\quad$ Yes & 0.87 & $0.81-0.93$ & $* *$ \\
No & & &
\end{tabular}

$\overline{\text { Based on 19,060 Medicaid Beneficiaries with newly diagnosed COPD }}$ obtained from Medicaid analytic extract files observed during 2005-2008. Asterisks represent significant group differences in COPD-specific healthcare utilization by statin therapy compared with non-users. Adjusted logistic regressions controlled for cohort year, sex, race, age, state, poverty eligibility, multimorbidity number of other clinical conditions, serious mental illness, alcohol, substance abuse, tobacco use, polypharmacy, and county variables, including above high school education (quartiles), unemployment density (quartiles), poverty density (quartiles), metro status, primary care shortage area, mental health shortage area, primary care provider density (quartiles), hospital beds density (quartiles), psychiatric hospital, pulmonologist density, and cardiologist density

$A O R$ adjusted odds ratio, $C I$ confidence interval, COPD chronic obstructive pulmonary disease

$* 0.01 \leq p<0.05$

$* * 0.001 \leq p<0.01$

$* * * p<0.001$

hospitalizations (AOR 0.83; 95\% CI 0.75-0.91) (Table 5). However, the association between short-term statin therapy and COPD-specific emergency room visits and COPDspecific outpatient visits remained insignificant. We also conducted sensitivity analyses using unadjusted IPTW (using weights that were not adjusted for the number of individuals receiving statins). The results from the sensitivity analyses were similar to those observed with the adjusted IPTW. They are not represented in tabular form but are available upon request.

\section{Discussion}

This retrospective observational study was conducted to evaluate the COPD-specific outcomes associated with statin therapy among individuals with newly diagnosed COPD in a real-world practice setting. Approximately $30 \%$ of
Table 4 Logistic regressions on chronic obstructive pulmonary disease-specific healthcare utilization among Medicaid beneficiaries with newly diagnosed chronic obstructive pulmonary disease $(n=19,060)$

\begin{tabular}{llll}
\hline & AOR & $95 \%$ CI & Significance \\
\hline COPD-specific hospitalizations & & & \\
MM and statin therapy & 0.82 & $0.70-0.97$ & $*$ \\
MM and no statin therapy & Reference & \\
No MM and statin therapy & 0.98 & $0.67-1.46$ & \\
No MM and no statin therapy & 1.17 & $0.98-1.39$ & \\
COPD-specific emergency room visits & & \\
MM and statin therapy & 0.90 & $0.81-0.99$ & $*$ \\
MM and no statin therapy & Reference & \\
No MM and statin therapy & 0.99 & $0.77-1.27$ & \\
No MM and no statin therapy & 1.17 & $1.05-1.31$ & $* *$ \\
COPD-specific outpatient visits & & & \\
MM and statin therapy & 0.85 & $0.78-0.92$ & $* * *$ \\
MM and no statin therapy & Reference & \\
No MM and statin therapy & 0.88 & $0.73-1.05$ & \\
No MM and no statin therapy & 0.95 & $0.87-1.03$ & \\
\hline
\end{tabular}

Based on 19,060 Medicaid beneficiaries with newly diagnosed COPD obtained from Medicaid analytic extract files observed during 2005-2008. Asterisks represent significant group differences in likelihood of COPD-specific healthcare utilization by statin therapy/multimorbidity interaction categories compared with the reference group (no statin and no multimorbidity) obtained from adjusted logistic regression analyses. Adjusted logistic regressions controlled for cohort year, sex, race, age, state, poverty eligibility, multimorbidity number of other clinical conditions, serious mental illness, alcohol abuse, substance abuse, tobacco use, polypharmacy, county level variables including: above high school education density (quartiles), unemployment density (quartiles), poverty density (quartiles), metro status, primary care shortage area, mental health shortage area, primary care provider density (quartiles), hospital beds density (quartiles), psychiatric hospital, pulmonologist density, and cardiologist density

$A O R$ adjusted odds ratio, $C I$ confidence interval, $C O P D$ chronic obstructive pulmonary disease, $M M$ multimorbidity

$* 0.01 \leq p<0.05$

$* * 0.001 \leq p<0.01$

$* * * p<0.001$

Medicaid beneficiaries with newly diagnosed COPD received statins during the baseline period. In this study, we found that even after controlling for baseline characteristics and observed selection bias using IPTW, beneficiaries receiving statins were $24 \%$ less likely to have COPD-specific hospitalizations, $19 \%$ less likely to have COPD-specific emergency room visits, and $14 \%$ less likely to have COPD-specific outpatient visits among individuals with newly diagnosed COPD. Our results are consistent with those of a handful of studies that found beneficial effects when evaluating the effects of statins in terms of COPD-related hospitalizations $[17,20]$. However, to the 
Table 5 Logistic regressions on chronic obstructive pulmonary disease-specific healthcare utilization among Medicaid beneficiaries with newly diagnosed chronic obstructive pulmonary disease; inverse probability treatment weight adjustment

\begin{tabular}{|c|c|c|c|}
\hline & $\mathrm{AOR}$ & $95 \% \mathrm{CI}$ & Significance \\
\hline \multicolumn{4}{|c|}{ COPD-specific hospitalizations } \\
\hline \multicolumn{4}{|c|}{ Duration of statin therapy } \\
\hline Long-term & 0.73 & $0.66-0.80$ & $* * *$ \\
\hline Short-term & 0.83 & $0.75-0.91$ & $* * *$ \\
\hline \multicolumn{4}{|l|}{ No use } \\
\hline \multicolumn{4}{|c|}{ COPD-specific emergency room visits } \\
\hline \multicolumn{4}{|c|}{ Duration of statin therapy } \\
\hline Long-term & 0.75 & $0.67-0.83$ & $* * *$ \\
\hline Short-term & 0.95 & $0.81-1.11$ & \\
\hline \multicolumn{4}{|l|}{ No use } \\
\hline \multicolumn{4}{|c|}{ COPD-specific outpatient visits } \\
\hline \multicolumn{4}{|c|}{ Duration of statin therapy } \\
\hline Long-term & 0.84 & $0.77-0.90$ & $* * *$ \\
\hline Short-term & 0.89 & $0.79-1.00$ & \\
\hline No use & & & \\
\hline
\end{tabular}

Based on 19,060 Medicaid beneficiaries with newly diagnosed COPD obtained from Medicaid analytic extract files observed during 2005-2008. Asterisks represent significant group differences in likelihood of COPD-specific healthcare utilization by duration of statin therapy compared with no statin use. Adjusted logistic analyses controlled for cohort year, sex, race, age, state, poverty eligibility, multimorbidity number of other clinical conditions, serious mental illness, alcohol, substance abuse, tobacco use, polypharmacy, county variables including: above high school education (quartiles), unemployment density (quartiles), poverty density (quartiles), metro status, primary care shortage area, mental health shortage area, primary care provider density (quartiles), hospital beds density (quartiles), psychiatric hospital, pulmonologist density, and cardiologist density

$A O R$ adjusted odds ratio, $C I$ confidence interval, $C O P D$ chronic obstructive pulmonary disease

$* 0.01 \leq p<0.05$

$* * 0.001 \leq p<0.01$

$* * * p<0.001$

best of our knowledge, this is the first study to examine the relationship between statin therapy and COPD-specific outcomes. Furthermore, this is the only study to assess the impact of duration of statin therapy among individuals with newly diagnosed COPD. Findings from this study indicate that a beneficial impact of statins is directly related to the duration of statin therapy. Statistically significant reductions in COPD-specific emergency room visits and outpatient visits were only observed among beneficiaries who had $\geq 120$ days of statin medication supply during the baseline period.

In addition, our study findings also revealed that statin therapy was associated with additional benefits for adults with COPD and multimorbidity (Table 4) compared with those with multimorbidity and no statin therapy. Such additional benefits could potentially be explained by the anti-inflammatory properties of statin therapy. Biologically, reductions in inflammatory biomarkers indicate statin therapy has anti-inflammatory properties [29, 30]. As COPD is becoming recognized as a disease of systemic inflammation with high levels of inflammatory biomarkers $[8,31,32]$, the results from our analyses suggest that the biological benefits of statins in reducing systemic inflammation may also reduce COPD-specific complications. Moreover, statins have been shown to improve outcomes among patients with cardiovascular comorbidities, which is a primary cause of mortality among patients with COPD. The association between COPD, systemic inflammation, and cardiovascular mortality is very important since the majority of patients with COPD die from cardiovascular causes, and lung function is a predictor of all-cause and cardiac-specific mortality [33]. Thus, the accepted benefits of statins in treating cardiovascular diseases can be linked to improvement in COPD-specific outcomes among patients with newly diagnosed COPD.

Findings from this study add to the existing evidence that suggest the beneficial impact of statins on improved lung function, COPD symptoms, and exacerbations [14-18]. Collectively, these findings warrant the evaluation of statins in RCTs to establish the efficacy and safety of statin use among individuals with COPD. Some RCTs that were ongoing when we conceptualized the study have recently been completed [34-36]. Findings from one of these large prospective multicenter RCTs evaluating the efficacy of simvastatin on reducing COPD exacerbations differed from the conclusions of our study [34]. This may be due to differences in the study population, as the trial included individuals with moderate to severe COPD aged between 40 and 80 years, whereas our study included patients with newly diagnosed COPD aged between 40 and 64 years. Moreover, the trial specifically evaluated the efficacy of simvastatin. Therefore, more robust evaluations of all statins is required in both RCTs and real-world practice settings with longer follow-up to confirm the beneficial effects of statins that we observed in our study. Indeed, a recent analyses of the STATins in COPD Exacerbations (STATCOPE) trial indicates deficiencies in the trial and warrants the need for further analyses of statins among patients with COPD eligible to receive statins [37].

\subsection{Strengths and Limitations}

This study is the first real-world observational analyses of clinical and economic outcomes associated with statin therapy among patients with newly diagnosed COPD in a US setting. The major strength of our study is the use of administrative claims data that enabled us to identify all the medical conditions using diagnosis codes. The data also 
consisted of medication information with NDCs and days of medication supply information, which helps in determining duration of statin therapy. Furthermore, our study examined a high-risk vulnerable population in terms of Medicaid enrollees. The current study differs from any prior analyses as it evaluated the effects of statin therapy on COPD-specific outcomes.

This study has some limitations common among observational studies based on claims data. For example, we were not able to control for COPD severity because laboratory data were unavailable. Our study population was determined using ICD-9-CM codes rather than the gold standard approach of using spirometry laboratory values. However, the sensitivity and specificity of ICD-9-CM codes to identify COPD has been established in previous studies. The study used data from only four states, which does not provide a full coverage of US Medicaid population. We also excluded dual Medicare-Medicaid and managed care enrollees from our population, which limits the overall generalizability. Finally, our study is only generalizable to patients with newly diagnosed COPD; further research is required to evaluate the effectiveness of statin therapy among individuals with prevalent COPD.

Despite these limitations, this is the first real-world observational study to comprehensively examine the relationship between statin medications and COPD-specific outcomes in a US population. Findings from this study warrant further clinical trial investigation of statin use among individuals with COPD.

\section{Compliance with Ethical Standards}

This research was presented as a poster at the International Society of Pharmacoeconomics and Outcomes Research 20th Annual International Meeting, and parts of this manuscript are posted online.

This study was approved by the Institutional Review Board at West Virginia University.

Funding Dr. Sambamoorthi was supported by the National Institute of General Medical Sciences of the National Institutes of Health under award number U54GM104942. The content is solely the responsibility of the authors and does not necessarily represent the official views of the National Institutes of Health.

Conflict of interest Mayank Ajmera, Chan Shen, and Usha Sambamoorthi have no conflicts of interest.

Open Access This article is distributed under the terms of the Creative Commons Attribution-NonCommercial 4.0 International License (http://creativecommons.org/licenses/by-nc/4.0/), which permits any noncommercial use, distribution, and reproduction in any medium, provided you give appropriate credit to the original author(s) and the source, provide a link to the Creative Commons license, and indicate if changes were made.

\section{References}

1. Global strategy for the diagnosis, management, and prevention of COPD: 2013 update. Global initiative for chronic obstructive pulmonary disease; 2013.

2. Matera MG, Calzetta L, Rinaldi B, Cazzola M. Treatment of COPD: moving beyond the lungs. Curr Opin Pharmacol. 2012;12(3):315-22.

3. Nussbaumer-Ochsner Y, Rabe KF. Systemic manifestations of COPD. Chest. 2011;139(1):165-73.

4. Vogelmeier CF, Wouters EFM. Treating the systemic effects of chronic obstructive pulmonary disease. Proc Am Thorac Soc. 2011;8(4):376-9.

5. Schnell K, Weiss CO, Lee T, Krishnan JA, Leff B, Wolff JL, et al. The prevalence of clinically-relevant comorbid conditions in patients with physician-diagnosed COPD: a cross-sectional study using data from NHANES 1999-2008. BMC Pulm Med. 2012;12:26.

6. Cazzola M, Ciaprini C, Page CP, Matera MG. Targeting systemic inflammation: novel therapies for the treatment of chronic obstructive pulmonary disease. Expert Opin Ther Targets. 2007;11(10):1273-86.

7. Cazzola M, Matera MG, Rogliani P, Page C. Treating systemic effects of COPD. Trends Pharmacol Sci. 2007;28(10):544-50.

8. Fabbri LM, Rabe KF. From COPD to chronic systemic inflammatory syndrome? Lancet. 2007;370(9589):797-9.

9. Baigent C, Keech A, Kearney PM, Blackwell L, Buck G, Pollicino $\mathrm{C}$, et al. Efficacy and safety of cholesterol-lowering treatment: prospective meta-analysis of data from 90,056 participants in 14 randomised trials of statins. Lancet. 2005;366(9493):1267-78.

10. Schönbeck U, Libby P. Inflammation, immunity, and HMG-CoA reductase inhibitors: statins as antiinflammatory agents? Circulation. 2004;109(21 Suppl 1):II18-26.

11. Lee J, Lee D, Kim E, Choe KH, Oh YM, Shim TS, et al. Simvastatin inhibits cigarette smoking-induced emphysema and pulmonary hypertension in rat lungs. Am J Respir Crit Care Med. 2005;172(8):987-93.

12. Lee T, Lin M, Chang N. Usefulness of C-reactive protein and interleukin- 6 as predictors of outcomes in patients with chronic obstructive pulmonary disease receiving pravastatin. Am J Cardiol. 2008;101(4):530-5.

13. Takahashi S, Nakamura H, Seki M, Shiraishi Y, Yamamoto M, Furuuchi M, et al. Reversal of elastase-induced pulmonary emphysema and promotion of alveolar epithelial cell proliferation by simvastatin in mice. Am J Physiol Lung Cell Mol Physiol. 2008;294(5):L882-90.

14. Keddissi JI, Younis WG, Chbeir EA, Daher NN, Dernaika TA, Kinasewitz GT. The use of statins and lung function in current and former smokers. Chest. 2007;132(6):1764-71.

15. Lawes CMM, Thornley S, Young Hopkins R, Marshall R, Chan WC, et al. Statin use in COPD patients is associated with a reduction in mortality: a national cohort study. Prim Care Respir J. 2012;21(1):35-40.

16. Blamoun AI, Batty GN, DeBari VA, Rashid AO, Sheikh M, Khan MA. Statins may reduce episodes of exacerbation and the requirement for intubation in patients with COPD: evidence from a retrospective cohort study. Int J Clin Pract. 2008;62(9):1373-8.

17. Huang C, Chan W, Chen Y, Chen TJ, Chou KT, Lin SJ, et al. Statin use and hospitalization in patients with chronic obstructive pulmonary disease: a nationwide population-based cohort study in Taiwan. Clin Ther. 2011;33(10):1365-70. 
18. Ishida W, Kajiwara T, Ishii M, Fujiwara F, Taneichi H, Takebe $\mathrm{N}$, et al. Decrease in mortality rate of chronic obstructive pulmonary disease (COPD) with statin use: a population-based analysis in Japan. Tohoku J Exp Med. 2007;212(3):265-73.

19. Frost FJ, Petersen H, Tollestrup K, Skipper B. Influenza and COPD mortality protection as pleiotropic, dose-dependent effects of statins. Chest. 2007;131(4):1006-12.

20. Mancini GBJ, Etminan M, Zhang B, Levesque LE, FitzGerald JM, Brophy JM. Reduction of morbidity and mortality by statins, angiotensin-converting enzyme inhibitors, and angiotensin receptor blockers in patients with chronic obstructive pulmonary disease. J Am Coll Cardiol. 2006;47(12):2554-60.

21. Søyseth V, Brekke PH, Smith P, Omland T. Statin use is associated with reduced mortality in COPD. Eur Respir J. 2007;29(2):279-83.

22. CMS. Medicaid analytic eXtract (MAX) general information. https://www.cms.gov/MedicaidDataSourcesGenInfo/07_MAX GeneralInformation.asp. Baltimore: Centers for Medicare and Medicaid Services, US Department of Health and Human Services; 2011.

23. Ajmera M, Shen C, Pan X, Findley PA, Rust G, Sambamoorthi U. Inhaled anticholinergic use and all-cause mortality among elderly Medicare beneficiaries with chronic obstructive pulmonary disease. Int J Chron Obstruct Pulmon Dis. 2013;8:287-94.

24. Halpern R, Baker CL, Su J, Woodruff KB, Paulose-Ram R, Porter $\mathrm{V}$, et al. Outcomes associated with initiation of tiotropium or fluticasone/salmeterol in patients with chronic obstructive pulmonary disease. Patient Prefer Adherence. 2011;5:375-88.

25. Ajmera M, Raval AD, Shen C, Sambamoorthi U. Explaining the increased health care expenditures associated with gastroesophageal reflux disease among elderly Medicare beneficiaries with chronic obstructive pulmonary disease: a cost-decomposition analysis. Int J Chron Obstruct Pulmon Dis. 2014;9:339-48.

26. Cooke CR, Joo MJ, Anderson SM, Lee TA, Udris EM, Johnson $\mathrm{E}$, et al. The validity of using ICD-9 codes and pharmacy records to identify patients with chronic obstructive pulmonary disease. BMC Health Serv Res. 2011;11:37.
27. Gershon AS, Wang C, Guan J, Vasilevska-Ristovska J, Cicutto L, To T. Identifying individuals with physician diagnosed COPD in health administrative databases. COPD. 2009;6(5):388-94.

28. Austin PC, Schuster T. The performance of different propensity score methods for estimating absolute effects of treatments on survival outcomes: a simulation study. Stat Methods Med Res. 2016;25(5):2214-37.

29. Forrester JS, Libby P. The inflammation hypothesis and its potential relevance to statin therapy. Am J Cardiol. 2007;99(5): 732-8.

30. Gross NJ. Novel antiinflammatory therapies for COPD. Chest. 2012;142(5):1300-7.

31. Barnes PJ, Celli BR. Systemic manifestations and comorbidities of COPD. Eur Respir J. 2009;33(5):1165-85.

32. Sevenoaks MJ, Stockley RA. Chronic obstructive pulmonary disease, inflammation and co-morbidity: a common inflammatory phenotype? Respir Res. 2006;7:70.

33. Mannino DM, Kiriz VA. Changing the burden of COPD mortality. Int J Chron Obstruct Pulmon Dis. 2006;1(3):219-33.

34. Criner GJ, Connett JE, Aaron SD, Albert RK, Bailey WC, Casaburi R, et al. Simvastatin for the prevention of exacerbations in moderate-to-severe COPD. N Engl J Med. 2014;370(23): 2201-10.

35. Effect of rosuvastatin therapy in patients with stable chronic obstructive pulmonary disease (RODEO). NCT00929734. Bethesda: US National Institutes of Health; 2014. http:// clinicaltrials.gov/ct2/show/NCT00929734. Accessed 20 August 2014.

36. Lovastatin as a potential modulator of apoptosis in chronic obstructive pulmonary disease (COPD). NCT00700921 (Internet). Bethesda: US National Institutes of Health; 2014. http:// clinicaltrials.gov/ct2/show/NCT00700921. Accessed 20 August 2014.

37. Mancini GB, Road J. Are statins out in the COLD? The STATCOPE Trial. Can J Cardiol. 2015;31(8):970-3. 